\title{
Treatment of Advanced Emphysema with Emphysematous Lung Sealant (AeriSeal ${ }^{\circledR}$ )
}

\author{
F.J.F. Herth ${ }^{\mathrm{a}} \quad$ D. Gompelmann ${ }^{\mathrm{a}} \quad$ F. Stanzel ${ }^{\mathrm{b}} \quad$ R. Bonnet ${ }^{\mathrm{c}} \quad$ J. Behr ${ }^{\mathrm{d}} \quad$ B. Schmidt \\ H. Magnussen ${ }^{f} \quad$ A. Ernst ${ }^{a}, g \quad$ R. Eberhardt ${ }^{a}$
}

${ }^{a}$ Thoraxklinik am Universitätsklinikum Heidelberg, Heidelberg, ${ }^{b}$ Lungenklinik Hemer, Pneumologie - Schwerpunkt Thorakale Endoskope, Hemer, ' Zentralklinik Bad Berka GmbH, Klinik für Pneumologie, Bad Berka, dMedizinische Klinik und Poliklinik I, Pneumologie, Klinikum Grosshadern der LMU, München, ${ }^{e}$ Medizinische Klinik mit Schwerpunkt Infektiologie und Pneumologie, Charité, Berlin, and ${ }^{\mathrm{f}}$ Zentrum für Pneumologie und Thoraxchirurgie $\mathrm{GmbH}$, Krankenhaus Grosshansdorf, Grosshansdorf, Germany; ${ }^{9}$ Interventional Pulmonology, Beth Israel-Deaconess Medical Center, Boston, Mass., USA

\section{Key Words}

Lung volume reduction - Emphysema therapy •

Bronchoscopic lung volume reduction · Interventional pulmonology $\cdot$ Emphysematous lung sealant

\begin{abstract}
Background: This report summarizes initial tests of an emphysematous lung synthetic polymer sealant (ELS) designed to reduce lung volume in patients with advanced emphysema. Objectives: The primary study objective was to define a therapeutic strategy to optimize treatment safety and effectiveness. Methods: ELS therapy was administered bronchoscopically to 25 patients with heterogeneous emphysema in an open-label, noncontrolled study at 6 centers in Germany. Treatment was performed initially at 2-4 subsegments. After 12 weeks, patients were eligible for repeat therapy to a total of 6 sites. Safety and efficacy were assessed after 6 months. Responses were evaluated in terms of changes from baseline in lung physiology, functional capacity, and health-related quality of life. Follow-up is available for 21 of 25 patients. Results: Treatment was well tolerated. There
\end{abstract}

were no treatment-related deaths (i.e. within 90 days of treatment), and an acceptable short- and long-term safety profile. Physiological and clinical benefits were observed at 24 weeks. Efficacy responses were better among Global Initiative for Chronic Obstructive Lung Disease (GOLD) stage III patients $[\mathrm{n}=14$; change in residual volume/total lung capacity $(\Delta R V / T L C)=-7.4 \pm 10.3 \% ; \Delta$ forced expiratory volume in $1 \mathrm{~s}\left(\Delta \mathrm{FEV}_{1}\right)=+15.9 \pm 22.6 \%$; change in forced vital capacity $(\Delta \mathrm{FVC})=+24.1 \pm 22.7 \%$; change in carbon monoxide lung diffusion capacity $(\triangle D L C O)=+19.3 \pm 34.8 \%$; change in 6-min walk test $(\triangle 6 \mathrm{MWD})=+28.7 \pm 59.6 \mathrm{~m}$; change in Medical Research Council Dyspnea $(\triangle M R C D)$ score $=-1.0 \pm 1.04$ units; change in St. George's Respiratory Questionnaire ( $\Delta \mathrm{SGRQ}$ ) score $=-9.9 \pm 15.3$ units] than for GOLD stage IV patients $\left(\mathrm{n}=7 ; \Delta \mathrm{RV} / \mathrm{TLC}=-0.5 \pm 6.4 \% ; \Delta \mathrm{FEV}_{1}=+2.3 \pm 12.3 \%\right.$; $\Delta \mathrm{FVC}=+2.6 \pm 21.1 \% ; \Delta \mathrm{DLCO}=-2.8 \pm 17.2 \% ; \Delta 6 \mathrm{MWD}=$ $+28.3 \pm 58.4 \mathrm{~m} ; \Delta \mathrm{MRCD}=0.3 \pm 0.81$ units; $\Delta \mathrm{SGRQ}=-6.7$ \pm 7.0 units). Conclusions: ELS therapy shows promise for treating patients with advanced heterogeneous emphysema. Additional studies to assess responses in a larger cohort with a longer follow-up are warranted.

Copyright $\odot 2011$ S. Karger AG, Basel

Felix J.F. Herth, MD, PhD

Pneumology and Critical Care Medicine, Thoraxklinik, University of Heidelberg Amalienstrasse 5, DE-69126 Heidelberg (Germany)

Tel. +496221 396 1200, Fax +496221 3961202

E-Mail Felix.Herth@thoraxklinik-heidelberg.de (c) $2011 \mathrm{~S}$. Karger AG, Basel

$0025-7931 / 11 / 0821-0036 \$ 38.00 / 0$ 


\section{Introduction}

Bronchoscopic approaches to achieve lung volume reduction have recently been explored as safer alternatives to lung volume reduction surgery (LVRS) for treating advanced emphysema $[1,2]$. While these techniques are associated with less morbidity and mortality than LVRS, the majority have demonstrated limited effectiveness. Endobronchial valves, which are designed to obstruct the proximal airways and cause absorption atelectasis, have not consistently produced volume reduction, physiological benefit or improvements in symptoms [3-6]. Airway bypass therapy, developed for the treatment of homogeneous emphysema, has been associated with transient improvements in vital capacity and dyspnea, but the procedure is complex, and benefits have not been durable [7-9].

A corporate-sponsored (Aeris Therapeutics, Woburn, Mass., USA) study on human subjects was recently completed. The study tested a synthetic polymer (emphysematous lung sealant; ELS) designed to produce durable volume reduction by blocking up airways and collateral ventilation pathways, and sealing the collapsed region. It was designed as a noncontrolled, open-label, multicenter dose escalation study to identify the optimal treatment strategy. Approximately 24 patients were designated for enrollment in 3 treatment groups ( $n=8$ each). Because the dose required to achieve therapeutic benefit was unknown at the start of the tests, all patients were offered retreatment after 12 weeks. Group 1 patients received initial therapy at 2 subsegments in 1 lobe, and were eligible for retreatment at 2-3 additional sites. Group 2 patients received initial therapy at 3 subsegments in 1 lobe, and were eligible for retreatment at 2-3 additional sites. Group 3 patients received initial therapy at 4 subsegments in 1 lobe, and were eligible for retreatment at 1-2 additional sites. Follow-up was performed at 12 and 24 weeks following completion of therapy. This report summarizes responses through week 24 for 21 of 25 patients.

\section{Materials and Methods}

\section{Enrollment Criteria}

All study participants had heterogeneous, upper lobe predominant emphysema, respiratory symptoms despite American Thoracic Society (ATS)/European Respiratory Society (ERS) recommended medical therapy, and were either not eligible for, or had refused, LVRS and lung transplantation. Study inclusion criteria required: (1) heterogeneous emphysema defined by CT imaging; (2) persistent symptoms [i.e. a baseline Medical Research Council Dyspnea (MRCD) score of $\geq 2$ ] despite medical therapy; (3) age
$>40$ years; (4) severe airflow limitation defined as a ratio of forced expiratory volume in $1 \mathrm{~s}\left(\mathrm{FEV}_{1}\right)$ to forced vital capacity (FVC) $<70 \%$ and an $\mathrm{FEV}_{1}<50 \%$ predicted; (5) hyperinflation [total lung capacity (TLC) $>100 \%$ predicted and residual volume $(\mathrm{RV})>135 \%$ predicted]; (6) absence of $\alpha_{1}$-antiprotease deficiency; (7) absence of clinically significant pulmonary hypertension, defined as a pulmonary systolic pressure $>45 \mathrm{~mm} \mathrm{Hg}$ (by cardiac echo and/or right heart catheterization), and (8) abstinence from smoking for $\geq 4$ months prior to enrollment. Patients determined to be at high risk of mortality for LVRS, with an $\mathrm{FEV}_{1}<20 \%$ predicted and a carbon monoxide lung diffusion capacity (DLCO) $<20 \%$ predicted, were also excluded from study participation [10].

\section{Study Conduct}

Patients received ELS therapy at up to 6 target sites during either a single treatment session or 2 treatment sessions. All participating institutions contributed at least 1 patient.

Fourteen patients had Global Initiative for Chronic Obstructive Lung Disease (GOLD) stage III chronic obstructive pulmonary disease (COPD) and 11 GOLD stage IV COPD. Eight patients ( 6 stage III and 2 stage IV) were enrolled in group 1, and received initial treatment at 2 subsegments in 1 lobe; 3 received a second treatment at 2 additional subsegments. Eight patients were enrolled in group 2 ( 3 stage III and 5 stage IV) and received initial treatment at 3 subsegments in 1 lobe; 2 received a second treatment at 3 additional subsegments. Nine patients were enrolled in group 3 (5 stage III and 4 stage IV) and received initial treatment at 4 subsegments in 1 lobe; none underwent repeat therapy.

The study protocol was reviewed and approved by the Bundesinstitut für Arzneimittel und Medizinprodukte (BfArM) via Deutsches Institut für Medizinische Dokumentation und Information (DIMDI) and national and local ethics committees. All study participants reviewed and signed informed consent forms before enrolling.

Screening evaluations included pulmonary function tests (spirometry, plethysmography and single-breath diffusing capacity measurements), a 6-min walk test (6MWT), echocardiography, electrocardiography, chest CT scan performed at full inspiration, clinical pathology (hematology, coagulation studies, and serum chemistry measurements), a modified MRCD questionnaire, and a St. George's Respiratory Questionnaire (SGRQ) health-related quality of life questionnaire. Pulmonary function tests were performed according to published ATS/ERS guidelines. CT images were generated according to a standard acquisition/reconstruction algorithm (spiral acquisition using a multidetector CT scanner with $1 \mathrm{~mm}$ collimation, a pitch of 1 , and $0.5 \mathrm{~mm}$ overlap) [11].

ELS treatments were performed in the endoscopy suite under general anesthesia (24 patients) or conscious sedation (1 patient) per investigator preference. All patients were admitted to the hospital for observation following treatment.

\section{Outcome Measures}

The primary endpoint of the study was RV/TLC ratio measured 3 months following final treatment. Efficacy was further assessed in terms of change from baseline at 3 and 6 months in postbronchodilator $\mathrm{FEV}_{1}$ and FVC, DLCO, 6MWT distance, MRCD score, SGRQ health-related quality of life total domain score, and change from baseline in RV/TLC at 6 months.

Safety was assessed in terms of the incidence of adverse events and serious adverse events throughout the study. 
Table 1. Baseline physiology and functional assessments of study participants

\begin{tabular}{lccc}
\hline & Entire cohort $(\mathrm{n}=25)$ & GOLD stage III $(\mathrm{n}=14)$ & GOLD stage IV $(\mathrm{n}=11)$ \\
\hline $\mathrm{FEV}_{1}$, liters & $0.92 \pm 0.28(30.1 \pm 7.7)$ & $1.09 \pm 0.21(35.4 \pm 4.3)$ & $0.67 \pm 0.18(22.2 \pm 4.2)$ \\
FVC, liters & $2.44 \pm 0.64(60.0 \pm 12.3)$ & $2.59 \pm 0.55(63.4 \pm 11.3)$ & $2.21 \pm 0.69(54.8 \pm 12.3)$ \\
RV, liters & $5.81 \pm 1.09(257.9 \pm 53.0)$ & $5.37 \pm 0.89(229.2 \pm 43.1)$ & $6.47 \pm 1.01(301.1 \pm 33.5)$ \\
TLC, liters & $8.44 \pm 1.24(136.7 \pm 19.6)$ & $8.18 \pm 0.99(128.8 \pm 19.0)$ & $8.83 \pm 1.45(148.6 \pm 14.4)$ \\
DLCO, mmol/min/kPa & $2.20 \pm 1.21(25.8 \pm 11.2)$ & $2.63 \pm 1.22(29.9 \pm 11.5)$ & $1.52 \pm 0.78(18.3 \pm 5.7)$ \\
MRCD, units & $2.76 \pm 0.78$ & $2.67 \pm 0.72$ & $2.90 \pm 0.88$ \\
6MWD, m & $293.6 \pm 83.9$ & $324.6 \pm 84.8$ & $247.1 \pm 59.9$ \\
\hline
\end{tabular}

Results in parentheses are percentages of predicted.

\section{The ELS Procedure}

Disease heterogeneity was assessed by each principal investigator in conjunction with his or her consulting radiologist using CT images obtained at full lung inflation. Qualification for study participation was based solely on the subjective clinical CT assessments of principal investigators and their staff. Additional quantitative CT analysis was performed by the sponsor's medical staff using computerized algorithms (Pulmonary Workstation Plus Software, VIDA Diagnostics, Iowa City, Iowa, USA) for investigational purposes. All participants had upper lobe heterogeneous disease, and received treatment in the upper lobes or superior segments of the lower lobes.

Therapy was administered with the bronchoscope in wedge position at the airway subsegment. ELS foam sealant was delivered through a single lumen catheter with its tip positioned $2 \mathrm{~cm}$ beyond the bronchoscope. Wedge position was maintained throughout delivery to prevent backflow into the airway. The foam sealant was prepared at the bedside from aqueous polymer solution and cross-linker. Polymer solution contains $2 \%$ aminated polyvinylalcohol in phosphate buffer. Cross-linker consists of dilute, buffered pentane $1-5$ dial. Cross-linker $(0.5 \mathrm{ml})$ was added to the polymer substrate $(4.5 \mathrm{ml})$ in a 20 - $\mathrm{ml}$ syringe to initiate polymerization, which proceeds over approximately $3 \mathrm{~min}$. The 5 - $\mathrm{ml}$ solution was mixed with $15 \mathrm{ml}$ of air to generate $20 \mathrm{ml}$ of foam sealant by passing the material back and forth through a stopcock between the syringes 10 times. $20 \mathrm{ml}$ of liquid foam sealant was injected over 10-20 s. Wedge position was maintained for $1 \mathrm{~min}$ following delivery to allow complete in situ polymerization. The scope was then repositioned at the next treatment site, and the procedure repeated until all treatments were completed.

Polymer components are compatible with commercially available bronchoscopes. No special precautions were required in handling or cleaning bronchoscopy equipment following treatment.

\section{Data Presentation and Statistical Analysis}

Efficacy outcomes at 12 and 24 weeks after treatment are compared with baseline values and reported as change from baseline. Statistical significance of the posttreatment change in primary endpoint (RV/TLC ratio at 12 weeks) was assessed by nonparametric testing (Mann-Whitney test). A significant change was defined as $\mathrm{p}<0.05$. Comparisons for secondary endpoints were performed by nonparametric testing (Mann-Whitney) and statistical significance was based upon $\mathrm{p}$ values subject to correction for multiple comparisons using the method of Bonferroni. Correlations between continuous variables were performed using the method of Pearson.

\section{Results}

Patient Enrollment, Demographics, and Medical

History

Seventeen of the 25 patients in this cohort were males. Ages ranged from 49 to 74 years (62.7 \pm 7.4 , median 66). All patients were prior smokers (43.8 \pm 17.7 pack-years, median 38 pack-years years). Nine patients were oxygen dependent. None were wheelchair bound or used noninvasive ventilator support.

Eleven patients were receiving short-acting inhaled $\beta$ agonists, 7 short-acting anticholinergics, 17 long-acting inhaled $\beta$-agonists, 19 long-acting anticholinergics, and 18 inhaled steroids. Six patients used theophylline preparations and 4 oral corticosteroids. One patient was listed for lung transplantation. Three had previously undergone endobronchial lung volume reduction procedures: 1 patient had received ablative vapor therapy 8 months prior to ELS therapy; 1 had undergone valve placement and subsequent removal 24 months before; 1 had undergone valve placement and subsequent removal 9 months before. Baseline physiology and functional characteristics of study participants are summarized in table 1 .

\section{Safety Results}

Procedural Safety

All patients tolerated the ELS procedure without significant problems. There were no serious procedural or immediate postprocedural complications. Specifically, there were no episodes of bleeding, pneumothorax, respiratory failure requiring ventilator support, instances in 
which conscious sedation had to be converted to general anesthesia or instances where the procedure was aborted because of clinical instability. Three patients experienced spillage of material from the administration site into the central airways. In all 3 instances, the material was cleared by suctioning through the bronchoscope without consequence. The average procedure time to complete treatment was $19.7 \pm 8.6 \mathrm{~min}$ (median $18.1 \mathrm{~min}$ ), and the duration of each treatment was $6.9 \pm 2.4 \mathrm{~min}$ (median $7.3 \mathrm{~min}$ ).

\section{Posttreatment Complications Associated with ELS}

Therapy

ELS treatment was routinely associated with a 'flulike' reaction beginning 8-24 h following treatment. All patients treated with ELS experienced some component of this reaction. The most common side effects were elevated inflammatory markers (sedimentation rate and/or C-reactive protein; $\mathrm{n}=25)$, dyspnea $(\mathrm{n}=25)$, fever $(\mathrm{n}=$ $22)$, leukocytosis $(n=21)$, infiltrate on chest radiograph $(\mathrm{n}=16)$, and chest pain $(\mathrm{n}=12)$. Symptoms were generally self-limited and resolved within 24-96 h with supportive care. Symptoms lasting more than $24 \mathrm{~h}$ were treated with a combination of nonsteroidal anti-inflammatory medications, acetaminophen, corticosteroids, and additional supportive care (i.e. bronchodilators, antibiotics) as indicated. Leukocytosis and elevated inflammatory indices resolved within 3-7 days.

There were no treatment-related deaths (defined as a death within 90 days of treatment), episodes of treatmentrelated respiratory failure requiring ventilator support of $>24$ h duration, pneumothoraces, empyemas, lung abscesses, pulmonary emboli, episodes of heart failure, cardiac ischemia or myocardial infarction, or cardiac arrhythmia requiring medical treatment during follow-up.

Among the 14 patients with GOLD stage III emphysema, there were 6 COPD exacerbations, 4 of which were treatment related. One was an acute posttreatment exacerbation (day 6 after treatment), that resulted in prolongation of initial hospitalization, 3 were subacute (days 34 , 37 and 51 after treatment), and 2 were subchronic (days 89 and 110 after treatment). All were medically significant and required hospitalization and treatment with corticosteroids and antibiotics. One GOLD stage III patient experienced pneumonia 61 days after treatment at a nontreatment site that required inpatient medical therapy. One GOLD stage III patient experienced a clinically significant decline in pulmonary lung function (i.e. $\Delta \mathrm{FEV}_{1}=-17 \%$ from baseline) at 24 weeks' follow-up.

Among the 11 patients with GOLD stage IV COPD, there were 4 COPD exacerbations requiring hospitaliza- tion, all treatment related. Two occurred in the periprocedural period on days 5 and 11, and 2 were subacute, presenting on days 34 and 37 after the procedure. One event required supplemental oxygen and admission to the intensive care unit. Two additional patients presented with nontreatment-related respiratory failure (days 116 and 159 following treatment) requiring hospitalization and intensive care support. There were 2 pneumonias (days 1 and 45 after treatment) both of which required hospitalization and resolved with treatment. One patient developed acute colitis 27 days following treatment that required surgical intervention and another sustained clinically significant depression 71 days after treatment that required hospitalization and medical intervention. One patient presented with mild hemoptysis 284 days following treatment, which resolved spontaneously. One patient with GOLD stage IV disease experienced a clinically significant $(-12 \%)$ decline in $\mathrm{FEV}_{1}$ from baseline at 24 weeks' follow-up.

\section{Efficacy Results}

Efficacy responses are summarized in figure 1 and in tables 2-4 for: (a) the entire cohort (fig. 1; table 2); (b) GOLD stage III patients (table 3), and (c) GOLD stage IV patients (table 4). Wherever possible, published minimal clinically important difference (MCID) criteria as set forth by the ERS/ATS Standards Committee were applied to define the number of patients achieving a clinically significant response to therapy [12].

Figure 1 and table 2 summarize results for the entire cohort. Mean improvements at 12 weeks' follow-up were observed in spirometry $\left(\Delta \mathrm{FEV}_{1}=+7.9 \pm 19.1 \% ; \mathrm{p}=0.07\right.$ and $\Delta \mathrm{FVC}=+6.6 \pm 23.7 \% ; \mathrm{p}=0.21)$, gas trapping $(\Delta \mathrm{RV} /$ $\mathrm{TLC}=-3.4 \pm 9.2 \% ; \mathrm{p}=0.09)$, symptoms $(\Delta \mathrm{MRCD}=-0.5$ \pm 1.21 units; $\mathrm{p}=0.06)$, quality of life ( $\Delta$ SGRQ total domain score $=-7.1 \pm 14.2$ units; $\mathrm{p}=0.05)$, and exercise capacity $(\Delta 6 \mathrm{MWD}=+35.6 \pm 66.7 \mathrm{~m} ; \mathrm{p}=0.02)$, although none reached statistical significance following correction for multiple comparisons. Improvements from baseline for most outcome measures at 24 weeks were greater than at 12 weeks: $\Delta \mathrm{FEV}_{1}=+10.0 \pm 19.8 \%(\mathrm{p}=0.03), \Delta \mathrm{FVC}=$ $+15.86 .6 \pm 22.2 \%(\mathrm{p}=0.004), \Delta \mathrm{RV} / \mathrm{TLC}=-4.7 \pm 9.5 \%$ $(\mathrm{p}=0.04), \Delta \mathrm{MRCD}=-0.4 \pm 1.20$ units $(\mathrm{p}=0.16), \Delta \mathrm{SGRQ}$ total domain score $=-7.5 \pm 14.4$ units $(\mathrm{p}=0.05)$, and $\Delta 6 \mathrm{MWD}=+24.6 \pm 58.9 \mathrm{~m} ; \mathrm{p}=0.08$. Only the improvement in FVC was statistically significant when corrected for multiple comparisons.

Table 3 summarizes efficacy responses among patients with GOLD stage III COPD $(\mathrm{n}=14)$. ELS therapy was associated with a statistically significant change from baseline in the study primary endpoint, RV/TLC ratio at 


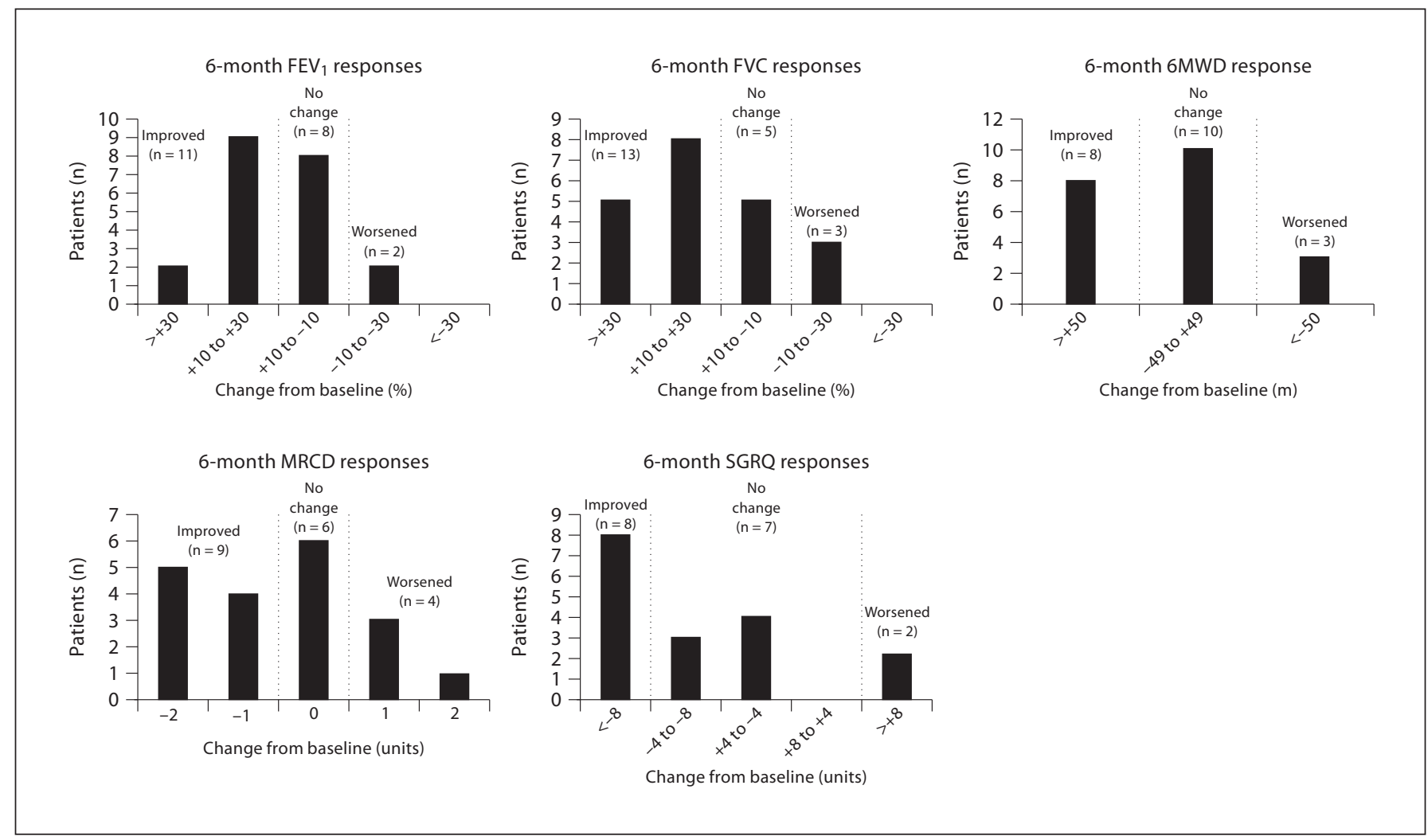

Fig. 1. Histograms showing response patterns 6 months following ELS therapy for those outcome measures with MCID criteria defining a clinically significant improvement. Criteria applied here are as follows: $\Delta \mathrm{FEV} \geq 10 \%$; $\Delta \mathrm{FVC} \geq 10 \% ; \Delta 6 \mathrm{MWD}>50 \mathrm{~m} ; \Delta \mathrm{MRCD}$ reduction $>1$ unit; $\Delta$ SGRQ reduction $>8$ units.

Table 2. Efficacy responses at 12 and 24 weeks' follow-up for GOLD stage III + IV patients

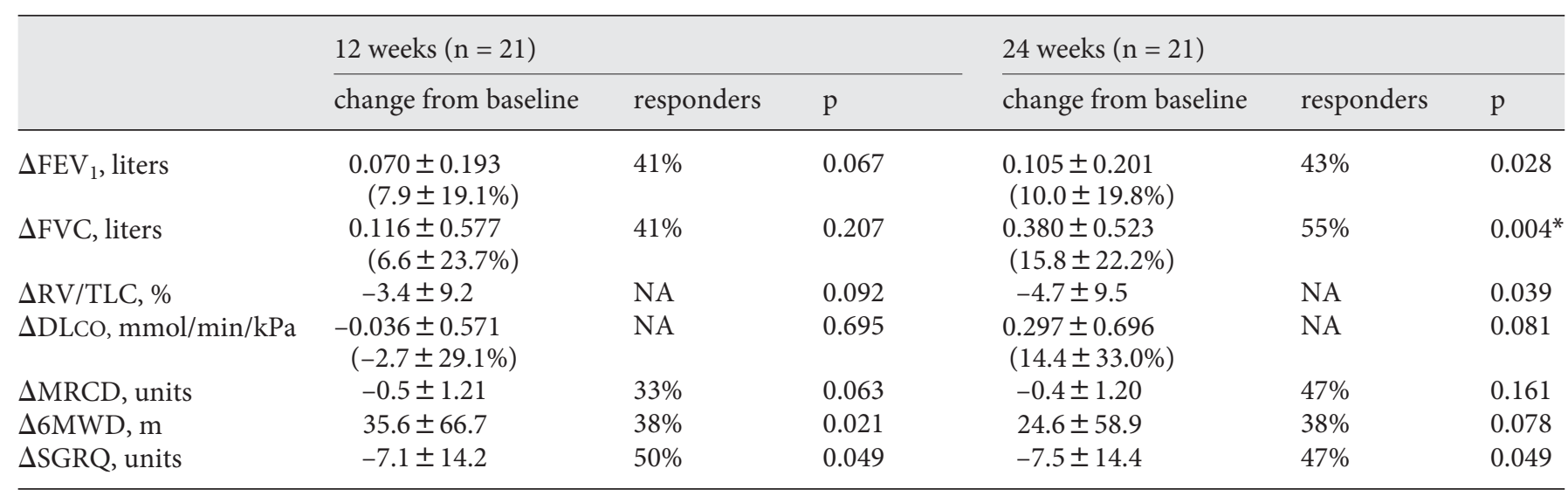

Responder criteria defined as follows: $\Delta \mathrm{FEV}_{1} \geq+15 \% ; \Delta \mathrm{FVC} \geq+15 \% ; \Delta \mathrm{MRCD} \leq-1$ unit; $\Delta 6 \mathrm{MWD} \geq 50 \mathrm{~m} ; \Delta \mathrm{SGRQ} \leq-8$ units; NA $=$ established MCID criteria do not exist. p values calculated compared to baseline. ${ }^{*}$ Denotes statistically significant change from baseline when corrected for multiple comparisons. 
Table 3. Efficacy responses at 12 and 24 weeks' follow-up for GOLD stage III patients

\begin{tabular}{|c|c|c|c|c|c|c|}
\hline & \multicolumn{3}{|l|}{12 weeks $(\mathrm{n}=14)$} & \multicolumn{3}{|l|}{24 weeks $(n=14)$} \\
\hline & change from baseline & responders & $\mathrm{p}$ & change from baseline & responders & $\mathrm{p}$ \\
\hline$\Delta \mathrm{FVC}$, liters & $\begin{array}{c}0.277 \pm 0.492 \\
(13.6 \pm 20.6 \%)\end{array}$ & $50 \%$ & 0.057 & $\begin{array}{c}0.495 \pm 0.465 \\
(24.1 \pm 22.7 \%)\end{array}$ & $64 \%$ & 0.010 \\
\hline$\Delta \mathrm{MRCD}$, units & $-1.0 \pm 1.18$ & $57 \%$ & 0.007 & $-1.0 \pm 1.04$ & $71 \%$ & 0.013 \\
\hline$\Delta 6 \mathrm{MWD}, \mathrm{m}$ & $41.3 \pm 74.8$ & $57 \%$ & 0.064 & $28.7 \pm 59.6$ & $36 \%$ & 0.106 \\
\hline$\Delta$ SGRQ, units & $-7.9 \pm 15.7$ & $50 \%$ & 0.105 & $-9.9 \pm 15.3$ & $67 \%$ & 0.048 \\
\hline
\end{tabular}

Responder criteria defined as follows: $\Delta \mathrm{FEV}_{1} \geq+15 \% ; \Delta \mathrm{FVC} \geq+15 \% ; \Delta \mathrm{MRCD} \leq-1$ unit; $\Delta 6 \mathrm{MWD} \geq 50 \mathrm{~m} ; \Delta \mathrm{SGRQ} \leq-8$ units; NA $=$ established MCID criteria do not exist. p values calculated compared to baseline. ${ }^{*}$ Denotes statistically significant change from baseline.

Table 4. Efficacy responses at 12 and 24 weeks' follow-up for GOLD stage IV patients

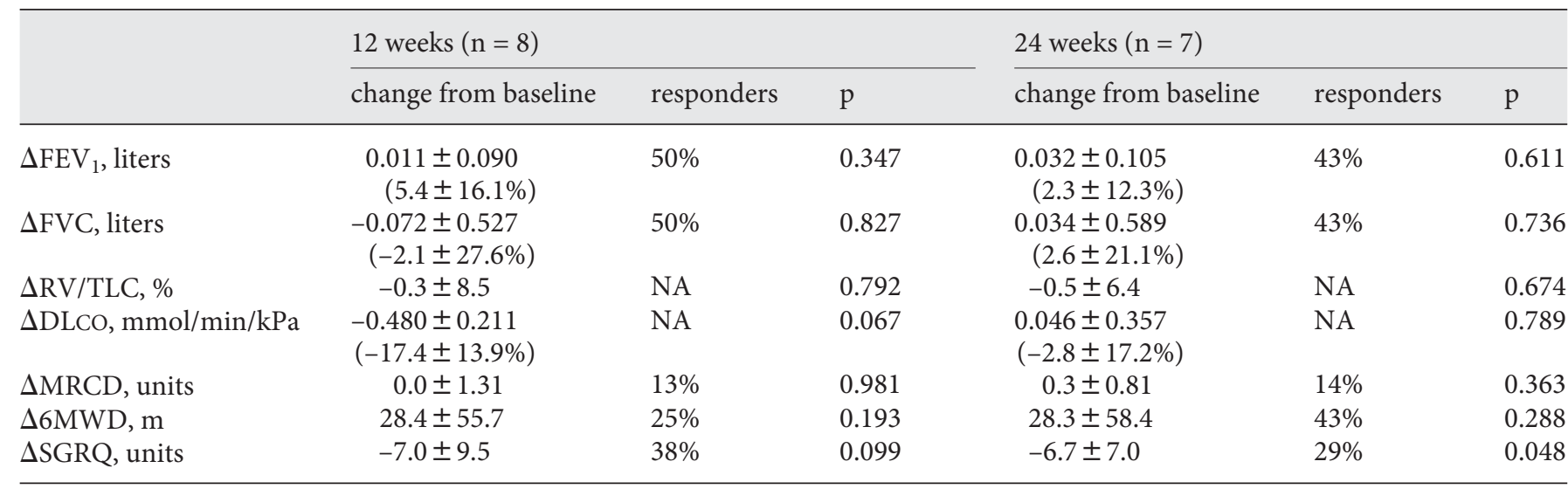

Responder criteria defined as follows: $\Delta \mathrm{FEV} 1 \geq+15 \% ; \Delta \mathrm{FVC} \geq+15 \% ; \Delta \mathrm{MRCD} \leq-1$ unit; $\Delta 6 \mathrm{MWD} \geq 50 \mathrm{~m} ; \Delta \mathrm{SGRQ} \leq-8$ units; NA $=$ established MCID criteria do not exist. p values calculated compared to baseline.

3 months' follow-up $(-5.4 \pm 8.6 \%, \mathrm{p}=0.035)$. Mean improvements in secondary efficacy outcome measures were observed at 3 and 6 months. Improvements from baseline at 3 months were observed in $\operatorname{FEV}_{1}(\Delta=+9.1 \pm$ $20.6 \%, \mathrm{p}=0.131)$, FVC $(\Delta=+13.6 \pm 20.6 \%, \mathrm{p}=0.057)$, $\operatorname{DLCO}(\Delta=+6.1 \pm 26.6 \%, \mathrm{p}=0.247), 6 \mathrm{MWD}(\Delta=+41.3$ $\pm 74.8 \mathrm{~m}, \mathrm{p}=0.064), \operatorname{MRCD}(\Delta=-1.0 \pm 1.18$ units, $\mathrm{p}=$ $0.007)$ and SGRQ total domain score $(\Delta=-7.9 \pm 15.7$ units, $\mathrm{p}=0.105)$. Improvements at 6 months were observed in $\operatorname{FEV}_{1}(\Delta=+15.9 \pm 22.6 \%, \mathrm{p}=0.048)$, FVC $(\Delta=+24.1 \pm 22.7 \%, \mathrm{p}=0.010), \mathrm{RV} / \mathrm{TLC}(\Delta=-7.4 \pm$
$10.3 \%, \mathrm{p}=0.031), \operatorname{DLCO}(\Delta=14.6 \pm 27.1 \%, \mathrm{p}=0.053)$, $6 \operatorname{MWD}(\Delta=+28.7 \pm 59.6 \mathrm{~m}, \mathrm{p}=0.106), \operatorname{MRCD}(\Delta=-1.0$ \pm 1.04 units, $\mathrm{p}=0.013)$ and SGRQ total domain scores $(\Delta=-9.9 \pm 15.3$ units, $\mathrm{p}=0.048)$. Only the improvement in FVC was statistically significant when corrected for multiple comparisons.

Table 4 summarizes efficacy responses among patients with GOLD stage IV COPD $(n=7)$. Mean improvements for all efficacy outcome measures in stage IV COPD patients were smaller and more variable than those of stage III patients. 

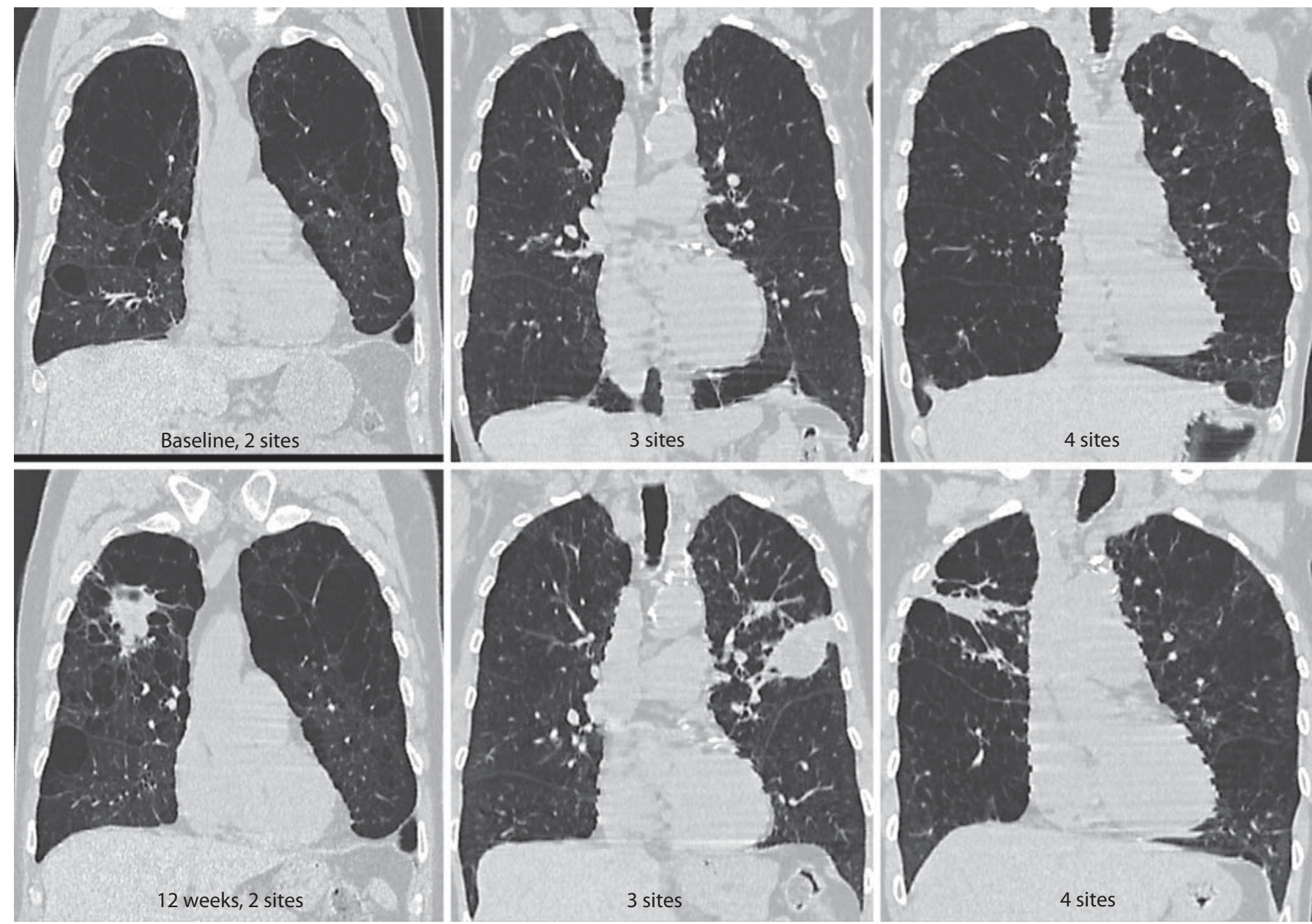

Fig. 2. Coronal CT images at baseline (top) and 12 weeks after ELS treatment (bottom) after 2-, 3- and 4-site therapy.

\section{Radiologic Results}

CT imaging was performed at baseline and 12 weeks after treatment to characterize the anatomic response to ELS therapy and the extent of lobar volume reduction at the sites of treatment. Baseline CT images confirmed the presence of moderate to severe heterogeneous emphysema. Parenchymal damage, characterized in terms of tissue density (Hounsfield units, HU) was as follows: (1) mean tissue density of both lungs $=-894 \pm 17 \mathrm{HU}$; (2) percentage of tissue less than $-950 \mathrm{HU}$ in the upper lung fields (right upper lobe + left upper lobe/2) $=52 \pm$ $10 \%$; (3) percentage of tissue less than $-950 \mathrm{HU}$ in the lower lung fields (right lower lobe + left lower lobe/2) = $39 \pm 12 \%$, and (4) the ratio of disease $<-950 \mathrm{HU}$ in the upper divided by lower lung field (\% <-950 upper/-950 lower $)=1.49 \pm 0.72$. Reduction in lobar vol- ume at 12 weeks' follow-up, expressed as decrease in gas volume per subsegment treated from analysis of CT images generated at full lung inflation, was $188 \pm 151 \mathrm{ml} /$ site [only 20 CT scans (14 GOLD stage III patients and 6 GOLD stage IV patients) were appropriately formatted to allow lobar volume assessments at 12 -week followup].

Representative CT images of patients treated at 2, 3 or 4 subsegmental sites are shown in figure 2 . Regional volume loss was observed at every treatment site across the cohort. ELS therapy was not associated with radiologic changes outside of treatment sites. There was no evidence of treatment-related mediastinal or pleural pathology. 


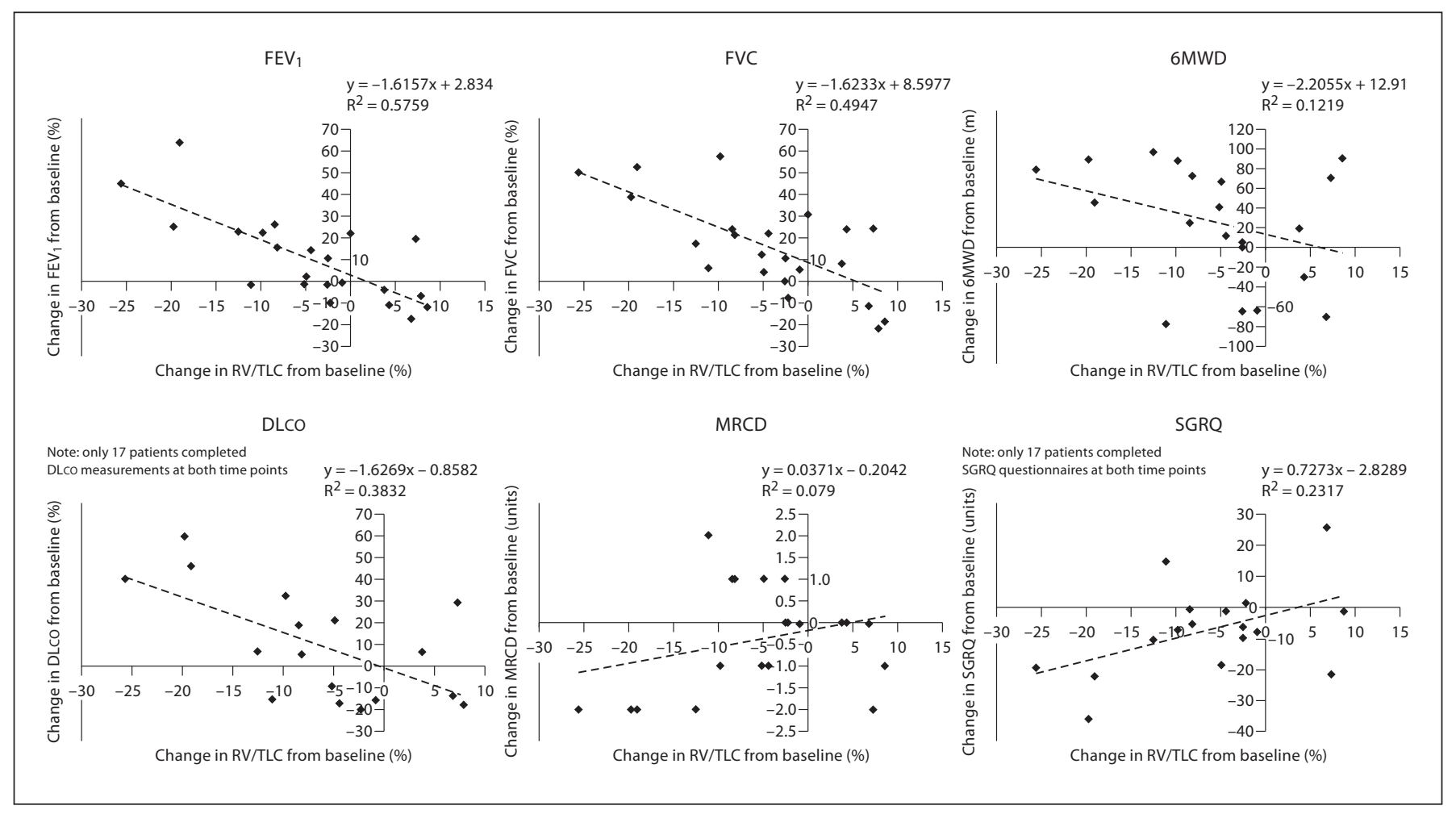

Fig. 3. Correlations between change in gas trapping and secondary outcome measures at 24 weeks. Correlations between $\triangle$ RV/TLC and secondary outcome measures 24 weeks following treatment for the entire cohort. Results show that changes in gas trapping correlated more closely with improvements in objective physiological outcome measures such as spirometry and diffusing capacity than with subjective outcome measures such as symptoms of dyspnea and quality of life.

\section{Correlation between Changes in Primary and Secondary Outcome Measures}

To assess the relationship between changes in gas trapping and changes in spirometry, gas exchange, symptoms, and quality of life following ELS therapy, \% $\Delta \mathrm{RV} /$ TLC from baseline was correlated with changes in $\mathrm{FEV}_{1}$, FVC, DLCO, 6MWD, MRCD, and SGRQ at 6 months' follow-up. Results, summarized in figure 3, show statistically significant correlations between reduction in RV/ TLC at 6 months, and improvements in $\mathrm{FEV}_{1}(\mathrm{r}=-0.759$, $p<0.001)$, FVC $(r=-0.703, p<0.001)$, DLCO $(r=-0.619$, $\mathrm{p}=0.012)$, and SGRQ total domain score $(\mathrm{r}=0.481, \mathrm{p}=$ $0.05)$.

\section{Discussion}

ESL (AeriSeal ${ }^{\circledR}$ System therapy) is a novel, minimally invasive alternative to LVRS being evaluated for treatment of patients with advanced emphysema. It appears to function by blocking small airways and collateral ventilation pathways, which leads to absorption atelectasis [13]. Results of this study indicate that ELS treatment can be performed safely in patients with advanced emphysema, and can reduce gas trapping and improve $\mathrm{FEV}_{1}, \mathrm{FVC}$, DLCO and quality of life up to 6 months in appropriately selected patients $[14,15]$.

Treatments were generally well tolerated, and did not require special equipment or fluoroscopy. Treatment times averaged approximately $7 \mathrm{~min}$ per site. Acute side effects consisted of an inflammatory 'flu-like' reaction that was observed in all patients, but was generally mild and responded to supportive medical care. Inflammatory reactions were, however, more pronounced in patients treated at 4 sites during a single session, and some patients in this cohort required additional supportive care and experienced longer hospitalizations than those treated at 2 and 3 sites.

There were no treatment-related deaths during the study. The most common serious complication associat- 
ed with ELS therapy was COPD exacerbation. Three periprocedural (with the first week of treatment), and 7 subacute (between post-treatment days 8 and 90) exacerbations were reported; the majority (8) were treatment related. The incidence of COPD exacerbations reported here is similar to that observed with other endobronchial lung volume reduction methods $[5,16,17]$. Late treatment-related complications ( $>90$ days after treatment), and evidence of sensitization following ELS treatment, such as eosinophilia, skin rash, or rhinitis were not observed.

Results show that ELS treatment reduces gas trapping, which correlates with improvements in pulmonary function, exercise capacity and quality of life. CT analysis indicates that ELS treatment causes local atelectasis at sites of treatment with reductions in gas volume at full lung inflation of approximately $190 \mathrm{ml}$ per treatment site. Overall responses at 12 and 24 weeks are similar to those previously reported following unilateral LVRS [18-21]. Physiological improvements following ELS therapy were greater in patients with GOLD stage III disease than stage IV disease. This is not unexpected given that stage IV patients had lower baseline spirometry (\% predicted $\mathrm{FEV}_{1}$ of $22.2 \pm 4.2$ vs. $35.4 \pm 4.3 ; \mathrm{p}<0.001$ ) and DLCO (\% predicted DLCO of $18.3 \pm 5.7$ vs. $29.9 \pm 11.5 ; \mathrm{p}<0.001)$, and greater hyperinflation (\% predicted RV of $301 \pm 34$ vs. $229 \pm 43 ; \mathrm{p}<0.001)$. These differences likely reflect more extensive tissue destruction and airway disease among the GOLD stage IV patients enrolled in this trial. Given the apparent mechanism of action of ELS, it is likely that for stage IV patients, additional volume reduction will be required to produce improvements in lung function equivalent to stage III patients.

Five patients underwent repeat ELS therapy in this study. Second sessions were well tolerated with no evidence of an exaggerated inflammatory reaction or allergic response. Additional clinical benefit, beyond that achieved with initial treatment, was observed in 2 of 5 patients. No patient experienced a decline in function following the second treatment session.

Among study patients $(n=3)$ who had received prior endobronchial volume reduction therapy (vapor ablation therapy in 1 patient, endobronchial valve therapy with subsequent valve removal in 2 patients), responses were variable. ELS therapy was associated with improved spirometry $\left(\Delta \mathrm{FEV}_{1}=+10.4 \%\right.$ and $+21.7 \% ; \Delta \mathrm{FVC}=+10.5 \%$ and $+30.8 \%)$, gas trapping $(\Delta \mathrm{RV} / \mathrm{TLC}=-2.5 \%$ and $-8.4 \%)$, and quality of life $(\Delta \mathrm{SGRQ}$ total domain score $=-9.8$ units and -17.2 units) in 2 patients (1 previously treated with vapor ablation, 1 previously treated with valves). The third patient, who had developed airway scarring and partial lobar collapse from prior valve treatment, worsened following ELS therapy $\left(\Delta \mathrm{FEV}_{1}=-17.4 \% ; \Delta \mathrm{FVC}=\right.$ $-11.5 \% ; \Delta \mathrm{RV} / \mathrm{TLC}=+6.8 \% ; \Delta \mathrm{SGRQ}$ total domain score $=$ +25.8 units). Thus, the potential utility of ELS therapy for patients who have failed prior endobronchial volume reduction therapy remains unclear.

While these initial results are encouraging, ELS therapy, in contrast to endobronchial valves, is not reversible. Thus selection of patient and target sites using guidelines similar to those for identifying LVRS candidates is necessary. Common indications for valve removal include airway complications associated with granulation tissue formation and bleeding, and postobstructive infections. Although complications of this type were not observed in this study, additional follow-up is needed in a larger cohort to fully assess whether irreversibility of treatment represents an important limitation of ELS therapy.

In summary, ELS therapy is a novel endobronchial system that utilizes a synthetic polymeric tissue sealant to produce volume reduction for treating lung hyperinflation in advanced emphysema. While this study is too small to draw final conclusions about safety and effectiveness, initial results using ELS are promising and support additional testing.

\section{Financial Disclosure and Conflicts of Interest}

Source of support for study: Aeris Therapeutics, Woburn, Mass., USA.

References

1 Hopkinson NS: Bronchoscopic lung volume reduction: indications, effects and prospects. Curr Opin Pulm Med 2007;13:125-130.

2 Ingenito EP, Tsai LW: Evolving endoscopic approaches for treatment of emphysema. Semin Thorac Cardiovasc Surg 2007; 19:181189.

- 3 Hopkinson NS, Toma TP, Hansell DM, Goldstraw P, Moxham J, Geddes DM, Polkey MI: Effect of bronchoscopic lung volume reduction on dynamic hyperinflation and exercise in emphysema. Am J Respir Crit Care Med 2005;171:453-460.

-4 Toma TP, Hopkinson NS, Hillier J, Hansell DM, Morgan C, Goldstraw PG, Polkey MI, Geddes DM: Bronchoscopic volume reduction with valve implants in patients with severe emphysema. Lancet 2003;361:931-933.

-5 Wan IY, Toma TP, Geddes DM, Snell G, Williams T, Venuta F, Yim AP: Bronchoscopic lung volume reduction for end-stage emphysema: report on the first 98 patients. Chest 2006;129:518-526.
44

Respiration 2011;82:36-45
Herth et al. 
-6 Yim AP, Hwong TM, Lee TW, Li WW, Lam S, Yeung TK, Hui DS, Ko FW, Sihoe AD, Thung KH, Arifi AA: Early results of endoscopic lung volume reduction for emphysema. J Thorac Cardiovasc Surg 2004;127: 1564-1573.

$>7$ Cardoso PF, Snell GI, Hopkins P, Sybrecht GW, Stamatis G, Ng AW, Eng P: Clinical application of airway bypass with paclitaxeleluting stents: early results. J Thorac Cardiovasc Surg 2007;134:974-981.

$>8$ Lausberg HF, Chino K, Patterson GA, Meyers BF, Toeniskoetter PD, Cooper JD: Bronchial fenestration improves expiratory flow in emphysematous human lungs. Ann Thorac Surg 2003;75:393-397; discussion 398.

9 Macklem PT, et al: Airway bypass: a new treatment for emphysema. Am J Respir Crit Care Med 2005;167:A726.

$>10$ National Emphysema Treatment Trial Research Group: patients at high risk of death after lung-volume-reduction surgery. N Engl J Med 2001;345:1075-1083.

- 11 Fishman A, Martinez F, Naunheim K, Piantadosi S, Wise R, Ries A, Weinmann G, Wood DE; National Emphysema Treatment Trial Research Group: A randomized trial comparing lung-volume-reduction surgery with medical therapy for severe emphysema. N Engl J Med 2003;348:2059-2073.
12 Cazzola M, et al: Outcomes for COPD pharmacological trials: from lung function to biomarkers. Eur Respir J 2008;31:416-468.

13 Fessler HE: Collateral ventilation, the bane of bronchoscopic volume reduction. Am J Respir Crit Care Med 2005;171:423-424.

14 Fessler HE, Scharf SM, Ingenito EP, McKenna RJ Jr, Sharafkhaneh A: Physiologic basis for improved pulmonary function after lung volume reduction. Proc Am Thorac Soc 2008;5:416-420.

15 Fessler HE, Scharf SM, Permutt S: Improvement in spirometry following lung volume reduction surgery: application of a physiologic model. Am J Respir Crit Care Med 2002;165:34-40.

16 de Oliveira HG, Macedo-Neto AV, John AB, Jungblut S, Prolla JC, Menna-Barreto SS, Fortis EA: Transbronchoscopic pulmonary emphysema treatment: 1-month to 24month endoscopic follow-up. Chest 2006; 130:190-199.
17 Wood DE, McKenna RJ Jr, Yusen RD, Sterman DH, Ost DE, Springmeyer SC, Gonzalez HX, Mulligan MS, Gildea T, Houck WV, Machuzak M, Mehta AC: A multicenter trial of an intrabronchial valve for treatment of severe emphysema. J Thorac Cardiovasc Surg 2007;133:65-73.

18 Kotloff RM, Tino G, Palevsky HI, HansenFlaschen J, Wahl PM, Kaiser LR, Bavaria JE: Comparison of short-term functional outcomes following unilateral and bilateral lung volume reduction surgery. Chest 1998;113: 890-895.

19 Keenan RJ, Landreneau RJ, Sciurba FC, Ferson PF, Holbert JM, Brown ML, Fetterman LS, Bowers CM: Unilateral thoracoscopic surgical approach for diffuse emphysema. J Thorac Cardiovasc Surg 1996;111:308-315; discussion 315-316.

20 Geiser T, Schwizer B, Krueger T, Gugger M, Hof VI, Dusmet M, Fitting JW, Ris HB : Outcome after unilateral lung volume reduction surgery in patients with severe emphysema. Eur J Cardiothorac Surg 2001;20:674-678.

$>21$ Argenziano M, Thomashow B, Jellen PA, Rose EA, Steinglass KM, Ginsburg ME, Gorenstein LA: Functional comparison of unilateral versus bilateral lung volume reduction surgery. Ann Thorac Surg 1997;64:321326; discussion 326-327. 